\title{
ERRATA
}

Artigo originalmente publicado com data de aprovação errada

\section{Seleção de isolados de Metarhizium anisopliae e Beauveria bassiana patogênicos a soldados de Atta bisphaerica e Atta sexdens rubropilosa em condições de laboratório}

Selection of Metarhizium anisopliae and Beauveria bassiana isolates pathogenic to Atta bisphaerica and Atta sexdens rubropilosa soldiers under laboratory conditions

\section{Alzimiro Marcelo Conteiro Castilho ${ }^{\mathrm{I}}{\text { Marcelo Elias Fraga }{ }^{\mathrm{I}}{ }^{*}}^{\text {Elen de Lima Aguiar-Menezes }}{ }^{\mathrm{III}}$ Carlos Alberto da Rocha Rosa ${ }^{\text {II }}$}

Para html ou PDF, acesse, respectivamente:

http://www.scielo.br/scielo.php?script=sci_arttext\&pid=S0103-84782010000600001\&lng=en\&nrm=iso\&tlng=pt ou

http://www.scielo.br/pdf/cr/v40n6/a627cr2459.pdf

We sugest that subscribers photocpy this correction and insert the copy at the appropriate place where the article originally appeared.

Sugerimos que uma fotocópia das correções seja inserida na página correta onde o artigo apareceu originalmente.

\footnotetext{
'Programa de Pós-graduação em Microbiologia Veterinária, Universidade Federal Rural do Rio de Janeiro (UFRRJ), Seropédica, RJ, Brasil.

"Departamento de Microbiologia e Imunologia Veterinária, Instituto de Veterinária, UFRRJ, Rodovia BR 465, km 7, 23890-000, Seropédica, RJ, Brasil. E-mail: fraga@ufrrj.br. *Autor para correspondência.

II'Departamento de Entomologia e Fitopatologia, Instituto de Biologia, UFRRJ, Seropédica, RJ, Brasil. 